\title{
Kann Christus sprechen? Eine postkoloniale Überlegung zur Christologie
}

\author{
Dominik Gautier
}

\begin{abstract}
This article reconstructs Gayatri Chakravorty Spivak's claim that Western intellectuals should not rely on concepts of subaltern groups being capable of speaking for themselves. Instead, intellectuals should self-critically examine their own thinkingin terms of how they reproduce conditions that prevent the "other" to speak. Based on the Christologies of Reinhold Niebuhr and James H. Cone the article shows that Christologies try evoke "confusion" regarding dominant (Christian) understandings of the self, the world, and God. Such an approach results in an important intersection between postcolonial critique and critical Christology. The article concludes with thoughts on how processes of religious education can be opened up for postcolonial reflection through introducing Christology and media art.
\end{abstract}

Christliche Theologie ist nicht nur verwickelt in die Nachwirkungen des kolonialrassistischen Diskurses, sondern hat auch an der Ausgestaltung ebendieses Diskurses mitgewirkt. So kann in der Formierung des Christentums durch die theologisch begründete Abgrenzung vom Judentum eine Quelle des wertenden Denkens in den Kategorien von "Wir" und "Nicht-Wir" gesehen werden, das in der Moderne - zum Teil säkularisiert - zu Antisemitismus und Rassismus ausgebaut wurde. ${ }^{1}$ Im Hinblick auf diesen Diskurs, der nicht zuletzt auch von Versuchen gekennzeichnet ist, Christus als „deutschen Helden“ oder Repräsentanten weißer Kultur auszugeben, fragt dieser Beitrag, inwiefern eine postkoloniale Revision der Rede von der herausfordernden Bedeutung Christi eine Ressource sein kann, die zur Schwächung des wertenden Systems beitragen kann, das in gewaltvoller Weise das "Wir" in das Zentrum stellt und die "Anderen“ zum Schweigen bringt.

Zunächst widmen sich die Ausführungen dem Nachvollziehen der postkolonialen Kritik an differenzstützenden Deutungsmustern, wie sie durch

1 Vgl. J. Kameron Carter, Race. A Theological Account, New York 2008, 3-5.

(C) DOMINIK GAUTIER, 2020 | DOI:10.1163/9789004417434_007

This is an open access chapter distributed under the terms of the CC BY-NC 4.o license 
Gayatri Chakravorty Spivak geäußert wurde. Die Frage, ob „Unterdrückte“ sprechen und sich politisch Gehör verschaffen können, verneint sie in herausfordernder Weise. Am Beispiel Indiens zeigt sie, wie „westliche“ Deutungsmacht es weiterhin ehemals Kolonisierten unmöglich macht, sich politisch Gehör zu verschaffen. Sie fordert demgegenüber eine dekonstruktive Intellektualität, die das hegemoniale Wissen „verstört“ und diejenigen, die als die „Anderen“ markiert werden, weniger am Sprechen hindert. Daraufhin versucht der Beitrag mit den Christologien Reinhold Niebuhrs und James H. Cones zu zeigen, wie die Forderung nach "Verstörung" vorherrschenden Wissens auch theologisch durchdacht werden kann. Beide Theologen, so soll dargestellt werden, fragen danach, ob Christus "sprechen“ kann und gehen davon aus, dass Christus durch seine politische Verfügbarmachung, genau wie die Kolonisierten, an Artikulation gehindert wird. In ihren Kontexten reformulieren sie die Christologie jeweils als herausfordernde, kritische Kraft gegenüber den vorherrschenden Verhältnissen und sehen die messianische Bedeutung Jesu gerade darin, dass er sich seinen Verfügbarmachungen entzieht und die Perspektiven anderen Lebens aufzeigt. Der Beitrag schließt mit Überlegungen zu einer postkolonialen religiösen Bildung, die sich gewaltvoller Deutungsmacht bewusst ist und - ausgehend von der Herausforderung der Christologie - nach Wegen sucht, diese Wissensgewalt zu dekonstruieren.

\section{Kann die Subalterne sprechen? Gayatri Chakravorty Spivaks Kritik epistemischer Gewalt}

In ihrem Aufsatz Can the Subaltern Speak? nimmt Gayatri Chakravorty Spivak die Deutungsmacht „westlicher" Intellektueller in den Blick, die sie als epistemische Gewalt bezeichnet. Ihre Kritik entzündet sich an jener Position, die behauptet, die ehemals Kolonisierten könnten für sich selber sprechen und sich damit politisch artikulieren. Diese Auffassung ist Spivak zufolge als eine Strategie anzusehen, mit der sich "westliche“ Intellektuelle aus der Verantwortung für die Wirkung ihrer eigenen Denkstrukturen ziehen. Indem die Verantwortung für politische Veränderung den „Anderen“ zugeschoben wird, bleibt die koloniale Idee "westlicher" erhabener Subjektivität unbearbeitet. $^{2}$

2 Vgl. Gayatri Chakravorty Spivak, Can the Subaltern Speak? Postkolonialität und subalterne Artikulation, übersetzt von Alexander Joskowicz und Stefan Nowotny, mit einer Einleitung von Hito StEYERL, Wien/Berlin 2008, 21-41. 
Spivak erklärt hingegen, dass diejenigen die durch die unabgeschlossene Geschichte des Kolonialismus marginalisiert werden, nicht sprechen können. Ihr geht es dabei um die Kritik an "westlicher" Deutungsmacht, welche die "Anderen" in gewaltvoller Weise zum Schweigen bringt. Um hervorzuheben, dass dieses Zum-Schweigen-Bringen politisch hervorgebracht wird, nimmt sie eine marxistisch-feministische Analyse vor: Sie zieht hierfür den von Antonio Gramsci geprägten und von indischen Historikerinnen der Subaltern Studies Group verwendeten Begriff der Subalternität heran. Subalterne sind nicht jene, die „lediglich“ unterdrückt sind, sondern Menschen, vor allem auch Frauen, die aufgrund herrschender Wissensverhältnisse sehr begrenzte oder überhaupt keine Sprachmächtigkeit erlangen können. ${ }^{3}$

Um ihre Kritik an epistemischer Gewalt zu veranschaulichen, führt Spivak das Beispiel der „Witwenverbrennung“ im Hinduismus an, bei der Ehefrauen mit dem Leichnam ihrer Ehemänner in das Feuer gehen. Diese Praxis diente sowohl dem hinduistischen Patriarchat als auch der britischen Kolonialmacht als Medium der politischen Artikulation. Das Patriarchat nutzte es dazu, das Geschlechtergefüge zu verherrlichen, wonach die Frau zur bedingungslosen Treue dem Mann gegenüber verpflichtet wird. Die Kolonialmacht, die diese Praxis unbedingt verhindern wollte, gab die „Witwenverbrennung“ als eine Tradition aus, der gegenüber "Zivilisierung“ nötig ist und verschaffte sich so eine Legitimationsgrundlage für die Durchsetzung britischer Interessen. In keinem der beiden Fälle wird aber die Stimme der Witwen gehört, die - aus welchen Gründen auch immer - in das Feuer gehen. Die Subalterne, so wird hier mit Spivak deutlich, kann aufgrund der vorherrschenden patriarchalen und kolonialen Deutungsmacht - aufgrund der epistemischen Gewalt - nicht sprechen. ${ }^{4}$ Als ein weiteres Beispiel für ihr Anliegen führt Spivak die Selbsttötung Bhuvaneswari Bhaduris an, die - dem indischen Unabhängigkeitskampf angeschlossen - mit einem politischen Mord beauftragt wurde und sich angesichts dieser Situation das Leben nahm. Um dem Verdacht entgegenzuwirken, es könnte sich bei ihrer Selbsttötung um die Konsequenz einer illegitimen Liebesbeziehung handeln, wartete sie bis zu ihrer Menstruation, die einen anderen Grund als eine gesellschaftlich geächtete Schwangerschaft für ihren Tod nahelegen müsste. Bhaduri bezog sich mit diesem Versuch, Widerstand zu artikulieren, sowohl auf die Tradition der Selbstopferung der Witwen als auch auf diejenige Tradition des weiblichen Kampfes für die Unabhängigkeit Indiens, wie sie in der Verehrung der weiblichen hinduistischen Gottheit Durga zum Ausdruck kommt. Bhaduris Artikulation wurde aber entweder als

3 Vgl. a.a.O., $42-65$.

4 Vgl. a.a.O., 80-103. 
Opfer - und damit als Konsequenz einer geächteten Beziehung - oder aber als gescheiterter Widerstand einer Unabhängigkeitskämpferin gelesen. Die Stimme Bhaduris, die sich bewusst zwischen den kolonialen oder patriarchalen Artikulationsmöglichkeiten zu erheben versucht, kann aufgrund der Vorherrschaft der Interpretationsmuster aber nicht gehört werden. ${ }^{5}$

Angesichts dieser diskursiv verhallenden Stimme der Subalternen fordert Spivak nun in einer vom Dekonstruktivismus geprägten Perspektive die Verantwortung Intellektueller ein. Wahrgenommene Verantwortung besteht für sie darin, die Deutungsmuster zu kritisieren, welche das Nicht-sprechenKönnen, die Subalternität der „Anderen“ hervorbringen. ${ }^{6}$ Im Anschluss an Jacques Derrida erklärt Spivak, dass Intellektuelle sich angesichts ihrer Partizipation an epistemischer Gewalt der eigenen Nicht-Universalität bewusst werden müssen: „Derrida markiert die Gefahr einer Aneignung des/ der Anderen durch Assimilierung, die in linker Kritik angelegt ist. [...] Er ruft [danach] [...] „die innere Stimme, die Stimme des anderen in uns, delirieren [zu] lassen“7. Das Denken verliert sich, wie Spivaks Beispiele zeigen, in vereindeutigenden Mustern, die gewaltvolle Verhältnisse von „Eigentlichen“ und „Fremden“ bekräftigen und das Sprechen der „Anderen“ unmöglich machen. Indem sich dieses Denken aber verwirren - „delirieren“ - lässt, kann es möglicherweise zu Unterbrechungen der Verhältnisse kommen, welche das NichtSprechen-Können der "Anderen“ produzieren. ${ }^{8}$

2

\section{Kann Christus sprechen? Die Christologien Reinhold Niebuhrs und James H. Cones - postkolonial gelesen}

Mit den folgenden Ausführungen soll gezeigt werden, dass die Christologie ein Potential birgt, diesen postkolonialen Impuls der Beunruhigung über die Partizipation an epistemischer Gewalt auch theologisch zu bedenken. Mit Reflexionen über die christologischen Arbeiten Reinhold Niebuhrs und James $\mathrm{H}$. Cones soll gezeigt werden, dass postkoloniale Kritik nicht etwas sein muss, das erst zur Theologie hinzukommt, sondern hilfreich dafür ist, neu über ein Grundmoment aller Theologie nachzudenken, nämlich über die irritierende Bedeutung Christi für theologisches Denken.

5 Vgl. a.a.O., 103-105.

6 Vgl. a.a.O., $105^{-106 .}$

7 A.a.O., 106 (Hervorhebung im Original).

8 Vgl. a.a.O., $105^{-106 .}$ 
Niebuhr wehrt sich in seinen Arbeiten gegen eine theologische Ethik, die sich, so seine Ansicht, ihrer selbst zu sicher ist. Wenn Christus mit guten Absichten von der liberalen theologischen Bewegung als Repräsentant absoluter Gerechtigkeit in Anspruch genommen wird, so erklärt Niebuhr mit Blick auf den US-amerikanischen Fortschrittsoptimismus, geht die irritierende Bedeutung Christi in Bezug auf ethisches Denken verloren. Die von Christus ausgehende Stimme - so ließe sich in Anlehnung an die postkoloniale Kritik Spivaks sagen - wird durch seine Verfügbarmachung unhörbar. Christus ist damit nicht mehr Christus, sondern das Spiegelbild partikularer Interessen. Niebuhr versteht die Figur Christi demgegenüber - ähnlich wie die von Karl Barth geprägte Dialektische Theologie - als ein "Nein“ Gottes zu menschlichen Vorstellungen von Gerechtigkeit, die Anspruch auf Absolutheit erheben. Aus diesem "Nein“ schließt Niebuhr nun aber eine Ermutigung, ein "Ja“, zu erneuertem - sehr viel stärker selbstkritischen - Engagement für relative Gerechtigkeit. Infolgedessen kritisiert er auch Christologien, die sich in die Betonung der Unverfügbarkeit Christi „zurückziehen“ und die Frage nach der Gestaltung eines weniger dominanten Zusammenlebens - auch aus Scheu vor dem Begehen neuer Fehler - ausklammern. ${ }^{9}$

Christus, der sich in Niebuhrs Denken jeder Verfügbarmachung entzieht, stellt gerade in dieser Weise eine bleibende ethische Herausforderung dar, die theologisches Denken entgegen ihrer selbstgerechten Erstarrung in Schwingung versetzen will. Die Figur Christi kann damit als das Moment der Verwirrung des Denkens verstanden werden, den Spivak stark macht. So wie Spivak mit der Aussage, dass die Subalterne nicht sprechen kann, die Dominanzverwicklung der Intellektuellen zu „verstören“ versucht, kann gesagt werden, dass Christus nicht sprechen kann. Hiermit kann die Figur Christi zur stetigen Revision von Konzepten des Zusammenlebens motivieren und die Versuche unterbrechen, derartige Konzepte zu universalisieren.

In dieser postkolonialen Perspektive der Herausforderung lässt sich auch der Ansatz schwarzer Theologie lesen, wie er von Cone vertreten wird. Cone nimmt eine Reformulierung von Christologie mit Blick auf schwarze USamerikanische Geschichte vor und kommt zu der Aussage, dass Jesus Christus schwarz ist. Wenn er Christus mit Schwarzsein verbindet, versteht Cone dies nicht einfach als eine Politisierung der Theologie. Eher zielt er damit auf eine theologische Korrektur der hegemonialen Theologie, die sich der herausfordernden Bedeutung Christi entledigt hat, um sich mit rassistischen Verhältnissen arrangieren zu können. Schwarzsein stellt für Cone vor dem

9 Vgl. Reinhold Niebuhr, The Nature and Destiny of Man. A Christian Interpretation, Band II. Human Destiny, New York 1941, 95-97. 
Hintergrund der Geschichte des Rassismus ein Symbol der Gewalt- aber auch Befreiungserfahrung dar. Wenn Jesus, der sich mit den Marginalisierten solidarisierte und selbst Opfer der Gewaltverhältnisse seiner Zeit wurde und zugleich als Auferweckter geglaubt wird, der Gottes befreiende Zuwendung verkörpert, in seiner herausfordernden Bedeutung "hörbar" gemacht werden soll, dann ist er unter den Bedingungen eines rassistischen Kontextes als Schwarzer zu denken. Mit dem Gedanken des Schwarzseins Christi kann nun alle Theologie an ihre Nicht-Universalität und ihre Verantwortung erinnert werden, Christus nicht als "fromme“ Idee zu denken, sondern als einen, der - solidarisiert mit denen, die von Rassismus verletzt sind - die bestehende Ordnung verwirrt: Er ist nun nicht mehr als identifiziert mit der dominanten Kultur zu denken, sondern als an den Orten präsent wahrzunehmen, wo sich die Gewalt dieser Kultur manifestiert. ${ }^{10}$

Folgt man Wolfhart Pannenbergs Interpretation der Theologie Cones, muss diese in die Nähe eines Erwählungsglaubens gerückt werden, der sich in gefährlicher Selbstgerechtigkeit verliert." ${ }^{11}$ Postkolonial betrachtet, kann dem nicht zugestimmt werden. Es geht Cone nicht um die Instrumentalisierung des Christentums für unreflektierte Identitätspolitik und darum, wie Pannenberg sagt, „[die] Sprache der traditionellen Christologie [...] entsprechend um[zu] funktionieren zu einer Symbolik für diese Zielsetzung." ${ }^{12}$ Cones Gedanken zielen darauf, die auch von Niebuhr ausgemachte, aufrüttelnde Bedeutung Christi konkreter kontextuell zur Sprache zu bringen und damit auch die Differenzordnung von „Eigentlichen" und "Anderen“ anzufragen. Er tut dies, indem er Christus als präsent nicht in weißer Normalität, sondern im schwarzen Widerstand denkt. Zwar darf nicht vergessen werden, dass Cone, wie J. Kameron Carter bemerkt, mit seinem Denken dem Differenzdiskurs verhaftet bleibt, reproduziert der Entwurf einer schwarzen Theologie doch die Differenz zwischen „Schwarz" und „Weiß“. ${ }^{13}$ Das Dilemma, dass nämlich jedes intellektuelle Bearbeiten von Ungleichheitsverhältnissen in ebendiesen verfangen bleibt, muss daher stetig reflektiert werden. Theologie darf nie den Anspruch darauf erheben, das letzte Wort zu haben, vergisst sie dann doch das "aufstörende" Moment, das mit der Christologie gegeben ist. Mit dem Ansatz Cones ist trotz aller Verfangenheit eine Theologie gegeben, die mit der Rede vom schwarzen Christus theologische Traditionen durcheinander bringt - und

10 Vgl. James H. Cone, A Black Theology of Liberation, New York $1970{ }^{4} 2015,125-130$.

11 Vgl. Wolfhart Pannenberg, Systematische Theologie, Band iII, Göttingen 1993, $562-563$.

12 A.a.O., 563.

13 Vgl. CARTER, Race, 192. 
eine Möglichkeit aufzeigt, wie in der Theologie eine von Spivak geforderte postkoloniale, konkret rassismuskritische Beunruhigung vorherrschenden Denkens artikuliert werden kann.

\section{Anders sprechen? Epistemischer Wandel und postkolonial reflektierte religiöse Bildung}

Der von Spivak hervorgehobene Impuls der Irritation, der vom Bewusstsein um die eigene Partikularität ausgeht und der auch christologisch bedacht werden kann, verweist auf die Notwendigkeit eines epistemischen Wandels, wie es María do Mar Castro Varela formuliert. Im Anschluss an Spivak erklärt Castro Varela, dass es bei einer postkolonialen Irritation von Bildungsprozessen nicht um didaktische „Kniffe“ geht. Diese dienen eher der Selbstinszenierung der Lehrenden. Es geht darum, dass „der Geist vielmehr beständig in Schwingung versetzt ${ }^{\prime \prime}{ }^{14}$ wird, sodass eine Verschiebung des Wissens stattfinden kann, das die Dynamiken des Zum-Schweigen-Bringens der „Anderen“ weniger befördert. Wichtig ist hierbei der Bezug auf das Wissen derjenigen, deren Perspektiven historisch kontinuierlich ausgeklammert werden. Dabei geht es nicht darum, diese Sichtweisen als die authentischen Gegenentwürfe zu vorherrschendem Wissen zu verstehen, sehr wohl aber darum, sie als Unterbrechungen von normativen Bildungsprozessen zu achten. Schließlich zielt Castro Varela mit Spivak auf das Bereiten eines stetigen epistemischen Wandels, der neben dem Wahrnehmen der Stimme der „Anderen“vor allem Selbstkritikund das kritische Bearbeiten von sozialen Privilegien zum Inhalt hat. Die Differenzordnung von "Wir" und "Nicht-Wir“ ist für die meisten Menschen weiterhin eine attraktive Wirklichkeitswahrnehmung, so bemerkt Castro Varela - und begründet dies mit dem nur gering wahrnehmbaren Widerstand gegen ebendiese Ordnung. Umso wichtiger, aber auch herausfordernder ist es, sich für das Herstellen von Räumen einzusetzen, in denen jenseits der Festlegung von Lernzielen der Dissens mit den herrschenden Unterscheidungen von „Eigentlichen“ und „Anderen“ erprobt werden kann. Der Dissens sollte dabei aber nicht um seiner selbst willen gesucht werden, sondern auf einen neuen Konsens, auf die Veränderung des Ganzen des Zusammenlebens zielen. ${ }^{15}$

14 María do Mar Castro Varela, Von der Notwendigkeit eines epistemischen Wandels. Postkoloniale Betrachtungen auf Bildungsprozesse, in: Thomas Geier/Katrin U. Zaвorowski (Hg.), Migration: Auflösungen und Grenzziehungen. Perspektiven einer erziehungswissenschaftlichen Migrationsforschung (Studien zur Schul- und Bildungsforschung 51), Wiesbaden 2016, 43-59, 54.

15 Vgl. a.a.O., $54-55$. 
Auch religiöse Bildung, sofern sie sich von dem Impuls, der von der Christologie ausgeht, verunsichern lässt, kann als eine Bildung verstanden werden, die sich selbstkritisch gegenüber gesellschaftlichen Verfestigungen von "Wir" und "Nicht-Wir" verhält. Sie zielt damit auch auf den epistemischen Wandel, der Platz macht für das Fragliche, Nicht-Sichere und Räume schafft, in denen die Stimmen wahrgenommen werden, die im Zuge der Subalternisierung zum Schweigen gebracht werden. Konkret bedeutet dies, wenn in Bildungsprozessen etwa die christologischen Entwürfe Niebuhrs und Cones eingebracht werden, dass es nicht das Ziel sein kann, diese Ansätze einfach zur Kenntnis zu nehmen, als ginge es um das Schließen von Wissenslücken. Vielmehr sollte die Beschäftigung mit irritierend-christologischen Entwürfen der Irritation auch Raum verschaffen, sodass Verhärtungen des Alltags religiöser Bildung befragt werden können. Zur Auseinandersetzung mit der Christologie, die über bloßes Kennenlernen hinausgeht, könnte beispielsweise die Frage treten, ob im Rahmen eingeschliffener religiöser Bildungsprozesse überhaupt wahrgenommen werden kann, was eine Herausforderung durch Christus überhaupt bedeuten kann.

Sowohl Niebuhrs Gedanken, welche die Kritik gut gemeinten gesellschaftlichen Engagements zum Thema haben, als auch Cones Arbeiten, die auf die Bearbeitung rassistischer Verhältnisse zielen, könnten daher zum Beispiel eine Beschäftigung mit dem Videokunstprojekt Wendelien van Oldenborghs zur Seite gestellt werden. In ihrer Installation mit dem Titel From Left ToNight (2015), das zwischen Dokumentar- und Spielfilm angelegt ist, verfolgt sie Aktivistinnen in Großbritannien in ihren Gesprächen über politischen Widerstand. Sie macht dabei die Frage nach der Sichtbarkeit von Marginalisierten zum Thema, die durch die vorherrschenden Deutungsmuster - die epistemische Gewalt verzerrt wahrgenommen oder gar unhörbar gemacht werden. Der Film beginnt mit der Anrede und Frage eines Aktivisten an die Filmemacherin, die, weil er direkt in die Kamera spricht, auch die Betrachtenden anruft:

They film me, they film me, they film me, they film me. And there I was seen. They made up a story from all the fragments and then I was charged, seen as charged! Now you film me, you film me, you film me - and then you make up your story. And then I will be what? Visible?16

16 Eigenes Transkript der Eröffnungsszene aus:WendeLIEn VAn OLDEn borG H, From Left To Night [2015], gezeigt im Oldenburger Edith-Russ-Haus für Medienkunst im Rahmen der Ausstellung The Fevered Specters of Art - Die fiebrigen Gespenster der Kunst vom 11. November 2016 bis zum 15. Januar 2017. Ein Teil des Projektes kann eingesehen werden unter: vimeo.com/216500061 [2. September 2019]. 
Diese Medienkunst und die Frage nach dem Wahrgenommen-Werden können zum Nachdenken darüber anregen, wie auch die Figur Christi immer wieder durch ihre Verfügbarmachung in ihrer herausfordernden Bedeutung zum Schweigen gebracht wird - etwa dann, wenn er als moralischer Garant eines bürgerlichen Lebensstils oder gar als weißer Held präsentiert wird. Darüber hinaus kann mit einer Diskussion über Christologie und Videokunst ein Gespräch über die lebensweltliche Bedeutung von Christologie angeregt werden, geht es doch, folgt man Niebuhr und Cone, bei christologischen Überlegungen nicht so sehr um dogmatische Spekulation als vielmehr um die Bedeutung der Figur Christi für die Gestaltung des Zusammenlebens. Letztlich gilt es, darüber nachzudenken, wie es dazu kommt, dass das „Wir“-„NichtWir"-Schema so attraktiv zu sein scheint, oder wie Eske Wollrad in ihren Überlegungen zu einer postkolonialen Theologie bemerkt, warum es so schwer ist, sich "Jesus Christus anders vorzustellen als Tom Cruise in Sandalen" ${ }^{17}$.

Bei dem Versuch, vorherrschende theologische Denkströme zu unterbrechen, etwa durch das Einbringen schwarzer Theologie, könnte mit dem Videokunstprojekt und der Frage nach Gesehen- und Gehört-Werden immer auch kritisch betrachtet werden, wie die Rezeption von Christologien verläuft. $\mathrm{Zu}$ achten wäre darauf, dass Affekte nicht unreflektiert bleiben - die Affekte der Ablehnung, die sich als Vorwurf des „umgekehrten Rassismus“ gegenüber schwarzer Theologie äußern können, die Affekte der Vereinnahmung, bei denen eine Identifikation mit schwarzer Theologie stattfindet, ohne eigene Privilegierungen zu hinterfragen oder die Affekte, die schwarze Theologie einfach als "bessere“ Theologie der "Anderen“ hochhalten und der produktiven Konfrontation damit aus dem Weg gehen. Es sollte darauf geachtet werden, dass der herausfordernde Charakter der Christologie im Blick bleibt, sodass es zur Beunruhigung von Lernprozessen kommen kann.

In diesen Momenten, in denen sich dieser Herausforderung gestellt wird, liegt die Möglichkeit eines anderen Sprechens, eines epistemischen Wandels, in dem herrschaftsförmige - auch theologische - Selbstverständlichkeiten angefragt werden, die dafür verantwortlich sind, dass Subalterne nicht sprechen können. Gegenüber diesen Verhältnissen ist theologische Verantwortungsübernahme gefordert, die - wie Spivak mit Blick auf gut

17 Eske Wollrad, Auf den Leib geschrieben. Postkolonial-feministische Theologie meets Disability Studies, in: Stefanie Schäfer-Bossert/Elisabeth Hartlieb (Hg.), Feministische Theologie - Politische Theologie. Entwicklungen und Perspektiven, Sulzbach/Taunus 2012, 153-165, 165 . 
gemeinte Beschwörungen einer anderen Politik sagt - „nicht mit Pauken und Trompeten verleugne[t] [werden] darf "18.

\section{Bibliographie}

\section{Literatur}

CArter, J. Kameron, Race. A Theological Account, New York 2008.

Castro Varela, María do Mar, Von der Notwendigkeit eines epistemischen Wandels. Postkoloniale Betrachtungen auf Bildungsprozesse, in: THomAs GEIER/ Katrin U. Zaborowski (Hg.), Migration: Auflösungen und Grenzziehungen. Perspektiven einer erziehungswissenschaftlichen Migrationsforschung, Studien zur Schul- und Bildungsforschung 51, Wiesbaden 2016, 43-59.

Cone, JAMEs H., A Black Theology of Liberation, New York $1970{ }^{4} 2015$.

Niebuhr, Reinhold, The Nature and Destiny of Man. A Christian Interpretation, Band II. Human Destiny, New York 1941.

Pannenberg, Wolfhart, Systematische Theologie, Band III, Göttingen 1993.

Spivak, Gayatri Chakravorty, Can the Subaltern Speak? Postkolonialität und subalterne Artikulation, übersetzt von Alexander Joskowicz und Stefan Nowotny, mit einer Einleitung von Hito SteyerL, Wien/Berlin 2008.

Wollrad, Eske, Auf den Leib geschrieben. Postkolonial-feministische Theologie meets Disability Studies, in: Stefanie SchäfEr-Bossert/Elisabeth Hartlieb (Hg.), Feministische Theologie - Politische Theologie. Entwicklungen und Perspektiven, Sulzbach/Taunus 2012, 153-165.

\section{Filmverzeichnis}

Oldenborg h VAn, Wendelien, From Left To Night [2015], gezeigt im Oldenburger Edith-Russ-Haus für Medienkunst im Rahmen der Ausstellung The Fevered Specters of Art - Die fiebrigen Gespenster der Kunst vom 11. November 2016 bis zum 15. Januar 2017. Ein Teil des Projektes kann eingesehen werden unter: vimeo.com/216500o61 [2. September 2019].

18 SPIVAK, Subaltern, 106. 